\title{
Anesthetic Management of a Patient with Deteriorated Cardiac Function Following Cardiopulmonary Resuscitation
}

\author{
TAMAKI NAGATA, YASUNORI MISHIMA*, TAKAHIKO ITO, MAIKO SAWADA, \\ TERUYUKI HIRAKI, NOBUYA HAMADA, NAO MIYAWAKI, \\ ASUKA ITO, SEIJI WATANABE \\ AND KAZUO USHIJIMA
}

Department of Anesthesiology, Kurume University School of Medicine, Kurume 830-0011 and

*Department of Anesthesia, Oita Sanai Medical Center, Oita 870-1151, Japan

Received 11 March 2010, accepted 7 May 2010

\begin{abstract}
Summary: A 73-year-old woman suffering from an abdominal aortic aneurysm (AAA), unstable angina, and low cardiac function (32\% of ejection fraction) was scheduled for abdominal aortic replacement and coronary artery bypass grafting. However, before the scheduled operation the patient fell into cardiopulmonary arrest with ventricular fibrillation due to rupture of the AAA. Immediate cardiopulmonary resuscitation (CPR) using epinephrine and electrical defibrillation restored the spontaneous circulation. Following CPR, a continuous high-dose dopamine infusion $(15 \mu \mathrm{g} / \mathrm{kg} / \mathrm{min})$ was initiated and emergent abdominal aortic replacement was performed. On arrival at the operating room, the patient showed serious hypotension, atrial fibrillation with multifocal ventricular premature contractions, and metabolic acidosis. Transesophageal echocardiography (TEE) suggested that the circulatory collapse might have resulted from diastolic dysfunction and deteriorated compliance of the left ventricular (LV) wall, possibly due to myocardial stunning induced by myocardial ischemia, and tachycardia induced by hypovolemia, both of which are influenced by high doses of catecholamine. We accordingly transfused adequate amounts of blood products and gradually decreased the infusion rate of dopamine to $4 \mu \mathrm{g} / \mathrm{kg} / \mathrm{min}$, while carefully monitoring blood pressure, central venous pressure, and TEE. By the end of surgery hemodynamic parameters had recovered to near normal levels. In post-resuscitated and hypovolemic patients, caution should be taken when administering high levels of exogenous catecholamines, which can induce myocardial stunning and circulatory collapse.
\end{abstract}

Key words cardiopulmonary resuscitation, catecholamine-induced myocardial stunning, diastolic dysfunction, transesophageal echocardiogram

\section{INTRODUCTION}

Although the initial success of cardiopulmonary resuscitation (CPR) is quite high, the majority of victims die within several days. The main cause of circulatory collapse following CPR is considered to be myocardial dysfunction [1]. Therefore, careful and quick management of deteriorated myocardium is essential in post-resuscitation cases. This report describes the anesthetic management of a patient with low cardiac function after CPR, who underwent emergent aortic replacement for the ruptured AAA.

All correspondence to: Yasunori Mishima, Department of Anesthesia, Oita Sanai Medical Center, 1213 Oaza-Ichi, Oita 870-1151, Japan. Tel: +81-97-5411311 Fax: +81-97-541-5218 E-mail: mishima_yasunori@kurume-u.ac.jp

Abbreviations: AAA, abdominal aortic aneurysm; ABP, arterial blood pressure; AF, atrial fibrillation; $\beta$-AR, $\beta$-adrenoreceptor; BE, base excess; CABG, coronary aortic bypass grafting; CPA, cardiopulmonary arrest; CPR, cardiopulmonary resuscitation; CVP, central venous pressure; DBP, diastolic blood pressure; ECG, electrocardiogram; EF, ejection fraction; HR, heart rate; LV, left ventricle; POD, postoperative day; ROSC, restoration of spontaneous circulation; SBP, systolic blood pressure; TEE, transesophageal echocardiogram; VF, ventricular fibrillation; VPC, ventricular premature contraction. 


\section{CASE REPORT}

A 73-year-old woman (height, $151 \mathrm{~cm}$; weight, 55 $\mathrm{kg}$ ) suffering from an AAA (diameter $8.4 \mathrm{~cm}$ ) and unstable angina was scheduled for abdominal aortic replacement and coronary artery bypass grafting (CABG). Preoperative examinations showed coronary artery stenosis (right coronary artery, 90\%; circumflex artery, $100 \%$; left anterior descending artery, 90\%), aortic root diameter; $37 \mathrm{~mm}$, left atrial dimension; $45 \mathrm{~mm}$, interventricular septum thickness; $12 \mathrm{~mm}$, posterior left ventricular (LV) wall thickness; $9 \mathrm{~mm}, \mathrm{LV}$ enddiastolic dimension; $55 \mathrm{~mm}$, LV end-systolic dimension; $47 \mathrm{~mm}$, fractional shortening; $14.5 \%$, an ejection fraction $(\mathrm{EF})$ of $32 \%$ and severe hypokinesis in the posterior to inferior myocardial wall motion.

Twenty days before the scheduled operation, the patient had sudden abdominal pain, which resulted in cardiopulmonary arrest (CPA) with ventricular fibrillation (VF) due to rupture of the AAA. Immediate CPR using a total amount of $2 \mathrm{mg}$ of intravenous epinephrine and electrical defibrillation with endotracheal intubation resulted in the restoration of spontaneous circulation (ROSC). Approximately $45 \mathrm{~min}$ after the ROSC, emergent surgery to repair the ruptured AAA was per- formed. On arrival at the operating room, a continuous infusion of high-dose dopamine $(15 \mu \mathrm{g} / \mathrm{kg} / \mathrm{min})$ was started. The patient showed hypotension (systolic blood pressure: $\mathrm{SBP}, 50 \mathrm{mmHg}$ ), tachycardia (heart rate: $\mathrm{HR}$, $128 \mathrm{bpm}$ ) with atrial fibrillation (AF), and multifocal ventricular premature contraction (VPC). Analysis of arterial blood revealed metabolic acidosis $\left(\mathrm{FIO}_{2}, 1.0\right.$; $\mathrm{pH}, 7.14 ; \mathrm{PaO}_{2}, 541$ torr; $\mathrm{PaCO}_{2}, 35$ torr; $\mathrm{HCO}_{3}^{-}, 11.4$ $\mathrm{mmol} / \mathrm{l}$; base excess (BE), $-16.5 \mathrm{mmol} / \mathrm{l}$ ) and $451 \mathrm{mg} /$ dl of glucose.

Tracheal intubation had already been performed, and anesthesia was induced with $7.5 \mathrm{mg}$ of midazolam and maintained with a gas mixture of oxygen, air, and $1 \%$ sevoflurane, in combination with continuous nicorandil and intermittent fentanyl and rocuronium as needed. The monitoring included electrocardiogram (ECG), $\mathrm{SpO}_{2}, \mathrm{P}_{\mathrm{ET}} \mathrm{CO}_{2}$, left radial arterial blood pressure $(\mathrm{ABP})$, central venous pressure $(\mathrm{CVP})$ via a catheter placed through the right internal jugular vein, and transesophageal echocardiography (TEE). The initial monitoring showed serious hypotension $(\mathrm{SBP}<50$ $\mathrm{mmHg}$ ), tachycardia with AF and VPCs, and elevated CVP $(>18 \mathrm{mmHg})$; TEE indicated that both atria were dilated, with a reduction in $\mathrm{LV}$ chamber size and an increase in wall thickness; the EF was calculated to be

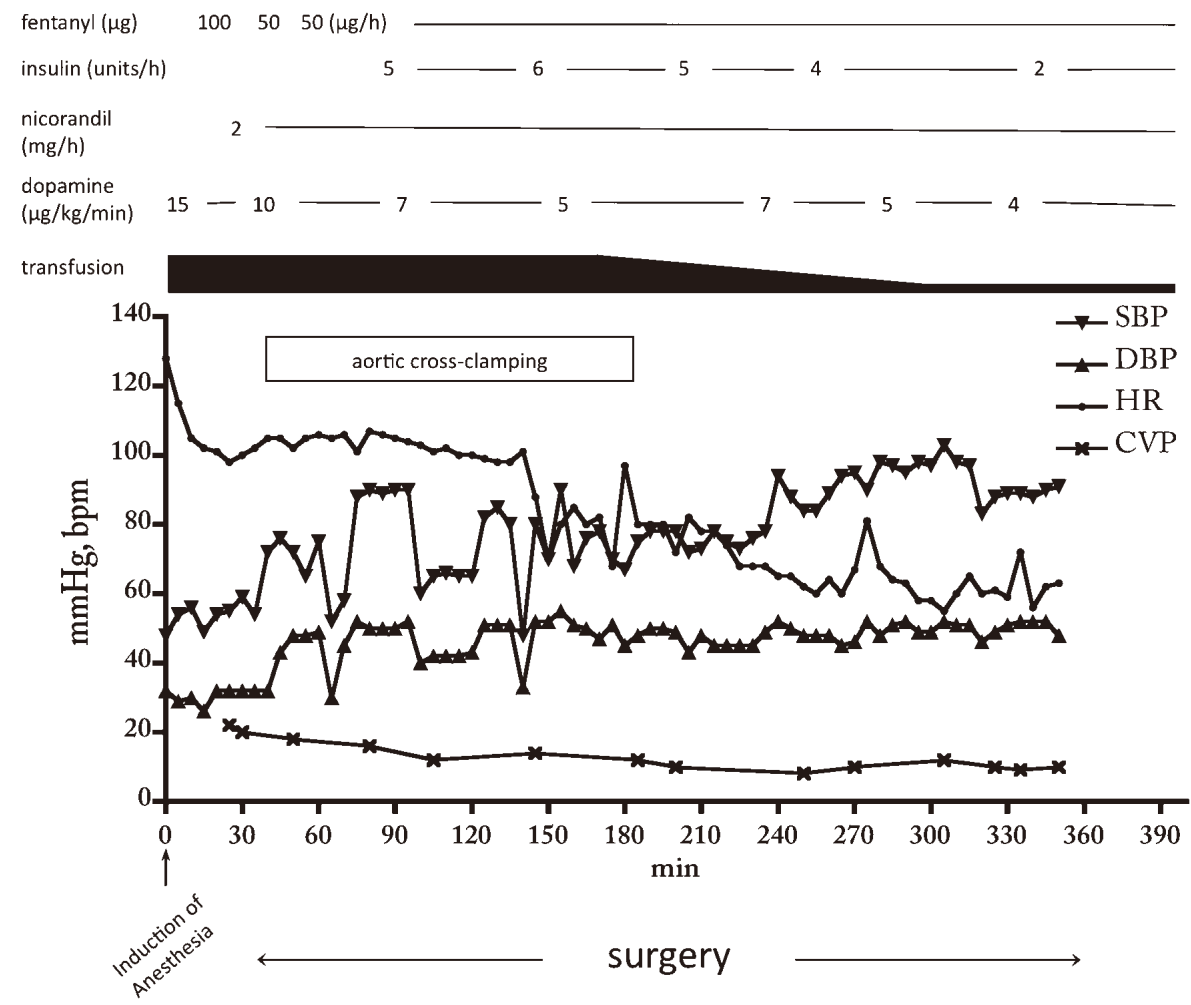

Fig. 1. Changes in hemodynamic parameters (SBP, DBP, HR and CVP) and doses of the anesthetics (sevoflurane and fentanyl), insulin, cardiovascular agents (nicorandil and dopamine) and blood transfusion products. 
$25 \%$. Furthermore, inferior- posterior wall motion was completely akinetic. Based on this data, we determined that the circulatory collapse was induced by diastolic dysfunction and deteriorated compliance of the LV wall. These conditions could have resulted from myocardial stunning induced by myocardial ischemia and tachycardia induced by hypovolemia; both conditions can be influenced by high doses of catecholamine. Based on these suppositions, the infusion rate of dopamine was decreased by degrees, and an adequate amount of blood products was transfused continuously under the careful monitoring of SBP, CVP, and TEE throughout the surgery (Fig. 1). The following blood products were transfused during the surgery against intraoperative $10,420 \mathrm{~g}$ of blood loss and $265 \mathrm{ml}$ of urine output: 34 units of packed red blood cells, 28 units of fresh frozen plasma, 20 units of concentrated platelets, and $1,750 \mathrm{ml}$ of crystalloidal or colloidal solutions. Consequently, SBP, HR, and CVP were steadily restored to normal limits, with TEE showing better LV contraction along with sufficient diastolic function. The ruptured AAA was uneventfully replaced just below the bilateral renal arteries and above the bifurcation of the common iliac artery in $295 \mathrm{~min}$. At the end of surgery, ABP, HR, CVP, EF, and cardiac output were $95 / 48 \mathrm{mmHg}, 66 \mathrm{bpm}, 10 \mathrm{mmHg}, 38 \%$, and 2.8 $1 / \mathrm{min}$, respectively, with the use of dopamine at an infusion rate of $4 \mu \mathrm{g} / \mathrm{kg} / \mathrm{min}$. The values of arterial blood analysis were as follows: $\mathrm{pH} 7.40, \mathrm{PaO}_{2} 323$ torr, $\mathrm{PaCO}_{2} 35$ torr, $\mathrm{HCO}_{3}{ }^{-} 21.5 \mathrm{mmol} / \mathrm{l}, \mathrm{BE}-2.3 \mathrm{mmol} / \mathrm{l}$ in $\mathrm{FIO}_{2}$ of $0.6, \mathrm{Hb} 10.2 \mathrm{~g} / \mathrm{dl}$, and glucose $154 \mathrm{mg} / \mathrm{dl}$ using a continuous venous insulin infusion. No ischemic change was observed and VPCs were almost completely absent on ECG throughout the procedure.

The patient was transferred to the intensive care unit, where mechanical ventilation was continued under sedation with propofol for 2 days, and the trachea was extubated on postoperative day (POD) 3 without any complications, including neurological deficits. However, difficulties in the management of unstable angina made it necessary for her to undergo $\mathrm{CABG}$ on POD 7, after which she made a remarkable recovery except for a minor surgical site infection.

\section{DISCUSSION}

Following CPR, many patients experience hemodynamic instability and can easily develop circulatory collapse, including hypotension, ventricular arrhythmias, and recurrent CPA, which can lead to early death even after initially successful ROSC. The major cause of circulatory collapse in the post-resuscitation period is considered to be myocardial stunning [1]. Our patient developed CPA because of myocardial ischemia following severe hypotension and ruptured AAA. ROSC was achieved by advanced cardiac life support involving the administration of epinephrine and electrical defibrillation, followed by a continuous infusion of high-dose dopamine. However, the patient fell into circulatory collapse after ROSC, probably due to diastolic dysfunction and deteriorated compliance of the $\mathrm{LV}$ wall. These LV conditions may have resulted from myocardial stunning induced by myocardial ischemia and administration of epinephrine during CPR accompanied by high doses of dopamine. The deteriorated diastolic dysfunction was deemed to be associated with rapid AF (induced by hypovolemia and high doses of catecholamine), and previous myocardial infarction. On the basis of SBP, HR, CVP, and TEE data, our priority was to restore hemodynamic function by improving LV wall compliance and increasing LV end-diastolic volume. We gradually decreased the infusion rate of dopamine while transfusing an adequate amount of blood products, which resulted in an improvement in cardiac dysfunction and hemodynamics.

Catecholamines are often given continuously during the post-resuscitation period in order to maintain hemodynamic stability. On the other hand, catecholamine-induced myocardial stunning can be caused by a surge in blood concentrations of catecholamines in association with the induction of $\beta$-adrenoreceptor ( $\beta$ AR). Catecholamines such as epinephrine, dopamine and dobutamine, especially in high doses, may increase myocardial oxygen consumption, resulting in an imbalance between myocardial oxygen supply and demand, which can produce myocardial stunning [2-8]. In our case, continuous infusion of high-dose dopamine had been initiated immediately following epinephrine administration for CPR, suggesting that these catecholamines might have exacerbated cardiac dysfunction in the post-resuscitation period. The effects of surged catecholamine levels on myocardial stunning and the induction of $\beta$-AR are reversible after catecholamine levels return to normal $[8,9]$. Some investigators have reported that blockade of $\beta$-AR significantly improves the initial outcomes of CPR, minimizes post-resuscitation myocardial dysfunction, and increases the duration of survival [7,10-12]. Although no $\beta$-AR blocking agents were administered in our case, stable cardiac function was obtained by reducing the infusion rate of dopamine while transfusing adequate volumes of blood products. Our present results suggest that excessive doses of catecholamine, as a $\beta$-AR agonist, induce myocardial stunning. 
Acidosis may impair cardiac resuscibility and reduce the myocardial response to catecholamines [13]. Acidosis should therefore be corrected to improve success in CPR and to allow for a reduction in the dose of catecholamines administered. In any case, the careful and speedy management of deteriorated myocardium is essential for patients after CPR, and appropriate monitoring of hemodynamics is of great importance in this regard. TEE may be very suggestive and is widely used to evaluate cardiac function, as shown in our case. This case report does have limitations. Although diastolic function is typically evaluated by means of transmitral valve blood flow (TMF) velocity, pulmonary venous blood flow (PVF) velocity, and tissue Doppler imaging (TDI), its evaluation by means of TMF and PVF in patients with AF is difficult. It is even more difficult to evaluate diastolic function in a critical situation, such as the case presented here. As a result, we are unable to present objective data, such as echocardiography, to demonstrate that high doses of dopamine suppressed diastolic function.

In summary, we have described the anesthetic management of a patient with CPA due to ruptured AAA and cardiac dysfunction following CPR. In post-resuscitation patients, particular attention should be paid to high levels of exogenous catecholamines, which can induce myocardial stunning and circulatory collapse.

\section{REFERENCES}

1. Xu T, Tang W, Ristagno G, Wang H, Sun $\mathrm{S}$ et al. Postresuscitation myocardial diastolic dysfunction following prolonged ventricular fibrillation and cardiopulmonary resuscitation. Crit Care Med 2008; 36:188-192.

2. Standards and guidelines for Cardiopulmonary Resuscitation (CPR) and Emergency Cardiac Care (ECC). National Academy of Sciences-National Research Council. JAMA 1986; 255:2905-2989.

3. Tang W, Weil MH, Sun S, Gazmuri RJ, and Bisera J.
Progressive myocardial dysfunction after cardiac resuscitation. Crit Care Med 1993; 21:1046-1050.

4. Tang W, Weil MH, Sun S, Noc M, Yang L et al. Epinephrine increases the severity of postresuscitation myocardial dysfunction. Circulation 1995; 92:3089-3093.

5. Vasquez A, Kern KB, Hilwig RW, Heidenreich J, Berg RA et al. Optimal dosing of dobutamine for treating post-resuscitation left ventricular dysfunction. Resuscitation 2004; 61:199-207.

6. McCaul CL, McNamara PJ, Engelberts D, Wilson GJ, Romaschin A et al. Epinephrine increases mortality after brief asphyxial cardiac arrest in an in vivo rat model. Anesth Analg 2006; 102:542-548.

7. Angelos MG, Butke RL, Panchal AR, Torres CA, Blumberg A et al. Cardiovascular response to epinephrine varies with increasing duration of cardiac arrest. Resuscitation 2008; 77:101-110.

8. Lyon AR, Rees PS, Prasad S, Poole-Wilson PA, and Harding SE. Stress (Takotsubo) cardiomyopathy: a novel pathophysiological hypothesis to explain catecholamine-induced acute myocardial stunning. Nat Clin Pract Cardiovasc Med 2008; 5:22-29.

9. Wittstein IS, Thiemann DR, Lima JA, Baughman KL, Schulman SP et al. Neurohumoral features of myocardial stunning due to sudden emotional stress. N Engl J Med 2005; 352:539-548.

10. Huang L, Weil MH, Cammarata G, Sun S, and Tang W. Nonselective beta-blocking agent improves the outcome of cardiopulmonary resuscitation in a rat model. Crit Care Med 2004; 32:S378-380.

11. Bassiakou E, Xanthos T, Koudouna E, Goulas S, Prapa V et al. Atenolol in combination with epinephrine improves the initial outcome of cardiopulmonary resuscitation in a swine model of ventricular fibrillation. Am J Emerg Med 2008; 26:578-584.

12. Cammarata G, Weil MH, Sun S, Tang W, Wang J et al. Beta1-adrenergic blockade during cardiopulmonary resuscitation improves survival. Crit Care Med 2004; 32:S440443.

13. Morimoto $\mathrm{Y}$, Kemmotsu O, and Morimoto $\mathrm{Y}$. Extramyocardial acidosis impairs cardiac resuscibility in isolated, perfused, rat hearts. Crit Care Med 1996; 24:17191723. 